\title{
Leitthema
}

Hautarzt 2013 - 64:26-31

DOI 10.1007/s00105-012-2409-9

Online publiziert: 28. November 2012

(c) Springer-Verlag Berlin Heidelberg 2012
P. Itin · D.A. Salgado

Dermatologie Universitätsspital Basel

\section{Genodermatosen, die der Praktiker kennen muss}

monogenen Erkrankungen mitbeteiligt ist, und über ein Drittel aller monogenen Erkrankungen zeigt eine Hautbeteiligung. Neben der Haut sind bei monogenen Erkrankungen besonders das muskuloskelettale Organ, Nervensystem und Augen mitbetroffen [3].

Die Genodermatosen sind heutzutage gut charakterisiert. Als wichtigste Informationsquelle sei die OMIM (Online Mendelian Inheritance in Man)-Datenbank erwähnt (http://www.ncbi.nlm. nih.gov/omim). Hier werden genetische Krankheiten katalogisiert und, soweit bekannt, den menschlichen Genen zugeordnet. Diese Datenbank basiert auf der Arbeit von Dr. Viktor A. McKusick et al. von der Johns Hopkins University von Baltimore. Sie wird regelmäßig mit den neusten Daten aktualisiert [4]. Jeder Eintrag in OMIM erhält zu seiner Katalogisierung eine Nummer zugeteilt. Diese MIM-Nummer besteht aus 6 Ziffern, wobei die erste Zahl den jeweiligen Erbgang der Erkrankung definiert. Die • Tab. 1 erläutert das Prinzip der MIM-Nummer.

Im Jahr 2008 wurden in OMIM 6016 monogene Erkrankungen aufgelistet, und davon waren 2310 genetisch vollständig aufgeklärt. Bei 1621 war der Erbmodus bekannt, und bei 2084 wurde der Erbmodus vermutet, und der Phänotyp war gut beschrieben. Im Jahr 2011 konnten bereits bei $>1000$ Krankheiten diagnostische Gentests durchgeführt werden. Genodermatosen stellen eine heterogene Gruppe von Erberkrankungen dar. Sie können in Verhornungsstörungen, Pigmentierungsstörungen, Gefäßbildungsstörungen, Bindegewebserkrankungen, Malignom-assoziierte Syndrome, Epidermolysen, Stoffwechselerkrankungen, fotosensitive Dermatosen, Immunmangelsyndrome und Erkrankungen der Haare und Nägel inklusive Schweißdrüsen und Talgdrüsen eingeteilt werden. Die ersten Zeichen und Symptome können bereits bei der Geburt oder in der frühen Kindheit auftreten oder sich auch erst im Erwachsenenalter bemerkbar machen. Die Genodermatosen verlaufen oft chronisch und können eine lebenslange Therapie notwendig machen. Die Vielzahl der unterschiedlichen Genodermatosen sind in der Regel sehr seltene Erkrankungen, die der nicht spezialisierte Dermatologe meist nicht kennt. Es gibt aber auch häufige Genodermatosen, die der niedergelassene Dermatologe kennen sollte (• Tab. 2). pischen Dermatitis weitere Realisationsfaktoren sein [2].

Genodermatosen sind erblich bedingte Krankheiten der Haut und ihrer Anhangsgebilde, die durch die Mutation eines einzelnen Gens entstehen. Gemäß WHO gibt es etwa 10.000 monogen vererbte Erkrankungen, wobei ca. 9500 autosomal und rund $500 \mathrm{x}$-chromosomal vererbt werden. Etwa 25 werden y-chromosomal gebunden vererbt, und 60 zeigen eine mitochondriale Heredität. Die Haut ist das Organ, das am häufigsten bei

\begin{tabular}{|lll}
\hline Tab. 1 & Erklärung der MIM-Nummern \\
\hline Erste Ziffer & MIM-Nummer & Erbgang \\
\hline 1 & $100000-199999$ & $\begin{array}{l}\text { Autosomal-dominanter Locus oder Phänotyp, vor dem } \\
15.05 .1994 \text { erstellt }\end{array}$ \\
\hline 2 & $200000-2999999$ & $\begin{array}{l}\text { Autosomal-rezessiver Locus oder Phänotyp, vor dem 15.05.1994 } \\
\text { erstellt }\end{array}$ \\
\hline 3 & $300000-399999$ & X-chromosomaler Locus oder Phänotyp \\
\hline 4 & $400000-499999$ & Y-chromosomaler Locus oder Phänotyp \\
\hline 5 & $500000-599999$ & Mitochondrialer Locus oder Phänotyp \\
\hline 6 & $600000-$ & Autosomaler Locus, nach dem 15.05.1994 erstellt \\
\hline
\end{tabular}




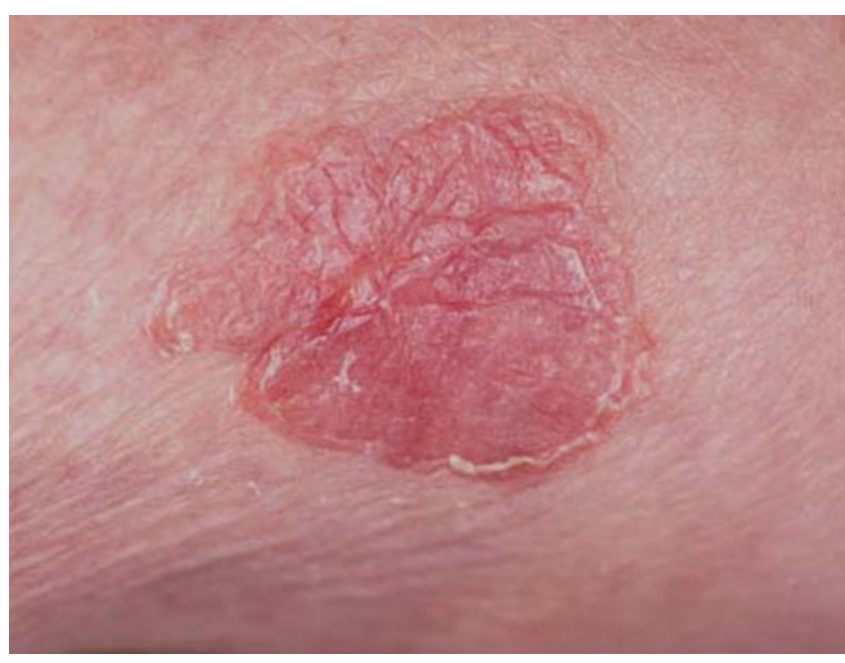

Abb. $1<$ Porokeratose

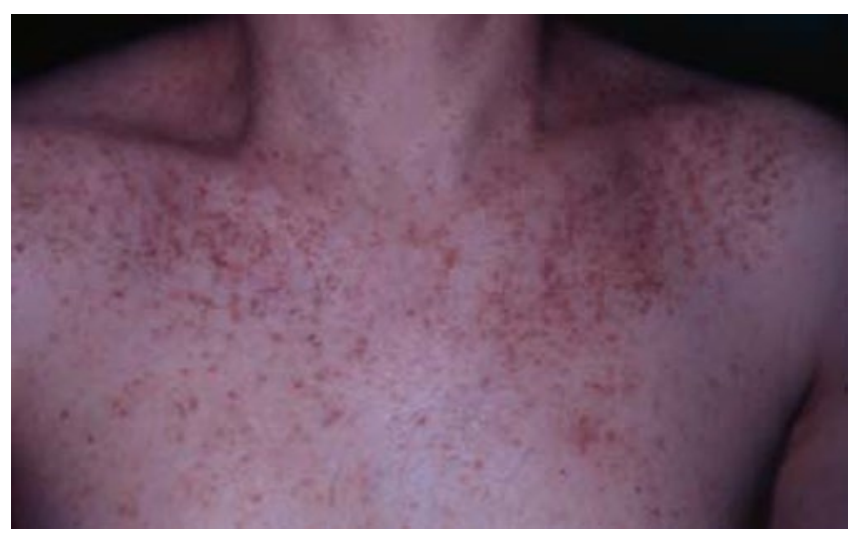

Abb. $3<$ Dyskeratosis follicularis Darier

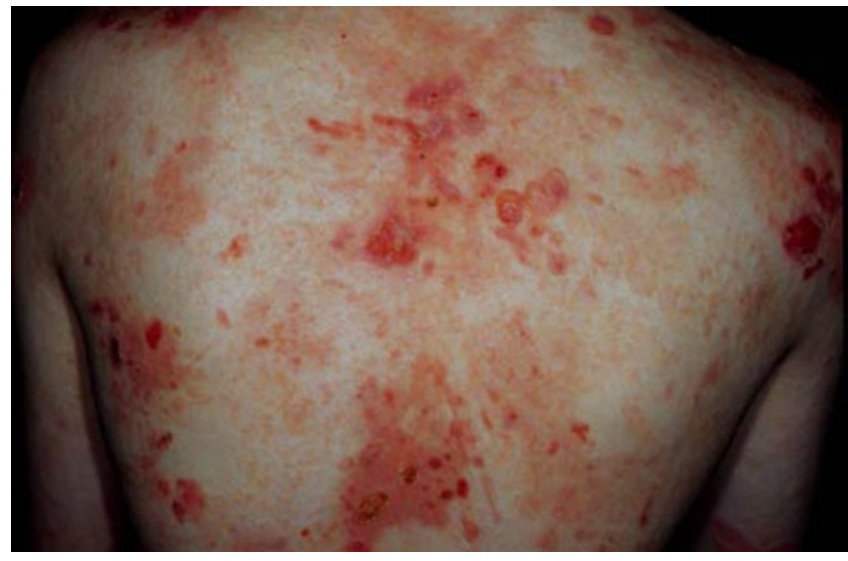

Abb. $5<$ Epidermolysis bullosa

\section{Wie erkennt man Genodermatosen?}

Zur Diagnose einer Genodermatose führen in erster Linie die klinische Untersuchung sowie die exakte Familienanamnese. Zur Bestätigung der Diagnose werden zum Teil Biopsien, Labor- und genetische Untersuchungen benötigt. Bei der eingehenden klinischen Untersuchung des
Patienten sollten insbesondere die Haut und ihre Anhangsgebilde, aber auch die Schleimhäute nach klinischen Merkmalen geprüft werden, denn ein Drittel aller monogenen Erkrankungen zeigt, wie bereits erwähnt, eine Beteiligung des Integuments. Die Familienanamnese kann dazu dienen, den möglichen Vererbungstyp zu erkennen und andere betroffene Familienmitglieder zu identifizieren.

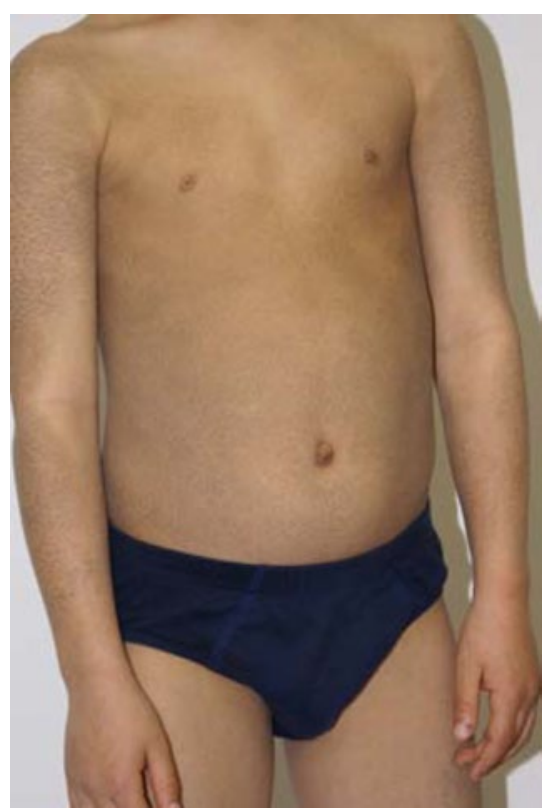

Abb. $2 \Delta$ Ichthyosis vulgaris

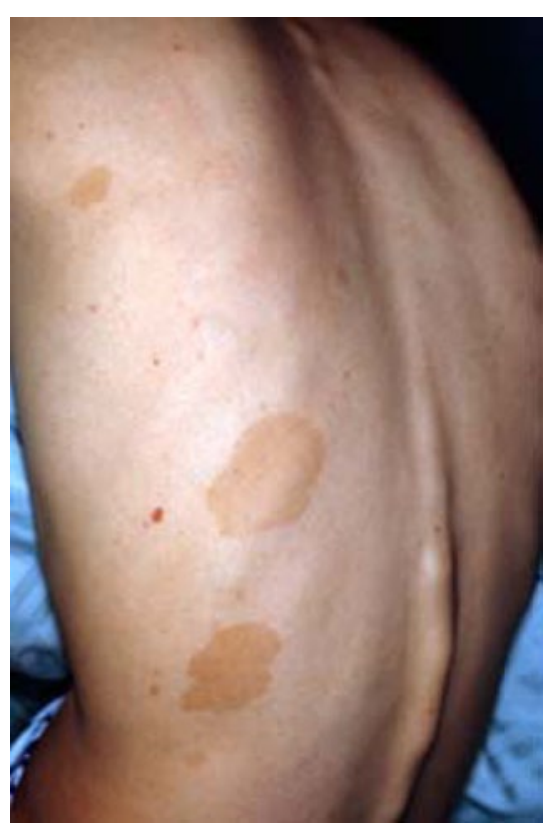

Abb. 4 \& Neurofibromatosis Recklinghausen

Eine Hautbiopsie bestätigt nicht selten eine klinische Verdachtsdiagnose.

Einzelne Genodermatosen haben typische Laborbegleitstörungen, und bei anderen können genetische Tests durchgeführt werden, falls die unterliegende $\mathrm{Mu}$ tation bereits beschrieben ist. 
Tab. 2 Diagnostik unserer 5 häufigsten

Genodermatosen

Genodermatosen Fälle \%

\begin{tabular}{|llll}
\hline 1 & Porokeratose & 43 & 38,0 \\
\hline 2 & Ichthyosis vulgaris & 23 & 20,3 \\
\hline 3 & Darier-Krankheit & 17 & 15,0 \\
\hline 4 & Neurofibromatose Typ 1 & 12 & 10,6 \\
\hline 5 & Epidermolysis bullosa & 9 & 7,9 \\
\hline & Total & 104 & 91,8 \\
\hline
\end{tabular}

\section{Material und Methoden}

Wir führten am Universitätsspital Basel, Klinik für Dermatologie, eine epidemiologische Studie durch, um die Häufigkeiten der einzelnen Genodermatosen an unserer Klinik zu bestimmen. Die Studie wurde vom lokalen Ethischen Komitee bewilligt. Als Datenquelle dienten die Diagnoselisten der gesamten ambulanten Konsultationen, die in der dermatologischen Universitätsklinik Basel im Zeitraum von Januar 2002 bis einschließlich Dezember 2008 erfasst wurden. Die Listen wurden in Excel-Tabellen erstellt und enthalten Angaben wie Konsultationsdatum, Geburtsdatum, Diagnose und den dazugehörigen ICD10-Code. In der Studie sind Patienten nicht berücksichtigt worden, bei denen primär keine Diagnose oder kein ICD10Code vermerkt wurde. Patienten mit ungültigen Codes sowie interne Konsilien wurden ebenfalls ausgeschlossen. Unter den insgesamt 48.354 berücksichtigten Patienten wurde bei 210 Personen eine Genodermatose diagnostiziert. Als Literatur- und Informationsquelle für die Recherchen über die Genodermatosen dienten in erster Linie folgende Internetseiten: - http://www.ncbi.nlm.nih.gov/omim,

- http://www.ncbi.nlm.nih.gov/pubmed,

- http://www.eurordis.org,

- http://www.orpha.net,

- http://www.genedx.com, emedicine. medscape.com

\section{Resultate}

Im Zeitraum zwischen 2002 und 2008 wurden insgesamt 48.354 ambulante Patienten berücksichtigt, davon waren 25.046 Frauen (52\%) und 23.308 Männer (48\%). Der Anteil der Frauen blieb über die ganze Zeit konstant über dem

Hautarzt 2013 - 64:26-31 DOI 10.1007/s00105-012-2409-9

(c) Springer-Verlag Berlin Heidelberg 2012

\section{P. Itin · D.A. Salgado \\ Genodermatosen, die der Praktiker kennen muss}

\section{Zusammenfassung}

Gesundheit oder Krankheit sind das Resultat von genetischer Konstellation und genetischer Veranlagung. Monogene Erkrankungen werden sehr stark durch die Mutation eines einzigen Gens phänotypisch geprägt. Gemäß WHO gibt es etwa 10.000 monogen verursachte Erkrankungen, und bei 1000 ist bereits eine molekulare Gentestung möglich. Genodermatosen im Zeitalter der digitalen Dokumentation sind heute gut charakterisiert, und die wichtigste genetische Datenbank kann über Google mit dem Stichwort "OMIM“ aufgerufen werden, welches eine Abkürzung für "Online Mendelian Inheritance of Man" darstellt. Hier werden genetische Krankheiten katalogisiert und, soweit bekannt, den menschlichen Genen zugeordnet. Wir prä- sentieren unsere eigenen epidemiologischen Daten aus der Dermatologie des Universitätsspitals Basel zu Genodermatosen. Es zeigte sich, dass folgende 5 monogenen Erkrankungen besonders häufig in der Praxis des Dermatologen gesehen werden: Porokeratosen, Ichthyosen, Darier-Krankheit, Neurofibromatosis Recklinghausen und Epidermolysis bullosa. Diese 5 Diagnosen machen $91 \%$ der Genodermatosen in der Sprechstunde eines Universitätsspitals aus.

\section{Schlüsselwörter}

Genodermatosen · Monogene

Erkrankungen · Molekular genetische

Diagnostik - Online Mendelian Inheritance of Man · Epidemiologie

\section{Important genodermatoses for the practitioner}

\section{Abstract}

Health or disease is a result of the genetic constellation and environmental influences. The phenotype of monogenic diseases is highly influenced by one single mutation. According to the WHO more than 10,000 monogenic diseases exist while for 1,000 diseases a molecular genetic test is available. Genodermatoses are well-documented and characterized; the most important data base for the diagnosis is the Online Mendelian Inheritance of Men data base, which can be searched in Google with the keyword "OMIM". Here genetic diseases are categorized and clinically described. We present our own epidemiolog- ic data from the Department of Dermatology, University Hospital Basel, concerning genodermatoses. Our results show that the most common genodermatoses seen in the daily practice are porokeratoses, ichthyoses, Darier disease, neurofibromatosis and epidermolysis bullosa. They account for $91 \%$ of all genodermatoses seen in a hospital-based dermatology department of Dermatology.

\section{Keywords}

Genodermatoses - Monogenic diseases .

Molecular genetic diagnosis - OMIM . Epidemiology
Männeranteil. Im untersuchten Zeitraum wurden 50 verschiedene Genodermatosen bei insgesamt 210 Patienten diagnostiziert (• Tab. 3). Im Jahr 2006 fanden sich 33 Patienten (15,7\%), 2003 waren es 29 Patienten (13,8\%), 2004 und 2008 jeweils 26 Patienten (12,3\%), 2002 wiederum 25 Patienten (11,9\%). Am wenigsten Patienten mit einer Genodermatose stellten sich im Jahr 2005 vor (20 Patienten, 9,5\%). Bei der Altersverteilung wurde die Gruppe der 0- bis 20-Jährigen in 3 Subgruppen geteilt. Dabei waren die 11- bis 20-Jährigen mit 11,1\% (23 Patienten) am meisten vertreten, gefolgt von den 0 - bis 5-Jährigen mit 10,6\% (22 Patienten) und den 6- bis 10-Jährigen mit 8,7\% (18 Patienten).

Von unseren 210 Patienten war die größte Gruppe jene der 41- bis 60-Jährigen mit 31,4\% (66 Patienten), gefolgt von der gesamten Gruppe der 0- bis 20-Jährigen mit 30,5\% (63 Patienten). Danach kamen die 21- bis 40-Jährigen mit 19,0\% (40 Patienten), die 61- bis 80-Jährigen mit $17,6 \%$ (37 Patienten) und schlussendlich die 81 - bis 100-Jährigen mit 1,4\% (3 Patienten). Die jüngste Patientin war eine Neugeborene mit Bloch-Sulzberger-Syndrom. Die älteste Patientin war 87 Jahre und hatte eine tuberöse Sklerose. In der Geschlechtsverteilung waren $64,8 \%$ Frauen (136 Patientinnen) und 35,2\% Männer 
(74 Patienten). Das Verhältnis Männer zu Frauen betrug also 1:1,8.

Insgesamt wurden 50 verschiedene Diagnosen gestellt, am häufigsten vertreten sind die erblichen Verhornungsstörungen mit 47,6\% (100 Patienten). Darunter bilden die Porokeratosen ohne nähere Angabe die größte Diagnosegruppe mit 43,8\% (43 Patienten), gefolgt von der Ichthyosis vulgaris mit 22,4\% (23 Patienten) und dem Morbus Darier mit 17,3\% (17 Patienten). Die nächste große Gruppe beinhaltet Genodermatosen mit Auftreten benigner Tumoren mit 14,5\% (30 Patienten). Hier ist die Neurofibromatosis Typ I die häufigste Diagnose mit 40\% (12 Patienten). Danach finden sich die erblichen Epidermolysen mit 11,4\% (24 Patienten), die erblichen Stoffwechselstörungen mit 6,2\% (13 Patienten), die Genodermatosen mit obligatem Auftreten maligner Tumoren und die sonstigen Genodermatosen mit je 5,2\% (11 Patienten), die erblichen Bindegewebserkrankungen mit 4,8\% (10 Patienten), die erblichen Pigmentstörungen mit 2,4\% (5 Patienten), die erblichen Krankheiten der Haare oder der Nägel mit 1,9\% (4 Patienten) und als Letztes die ektodermalen Dysplasien mit 0,9\% (2 Patienten).

\section{Die 5 häufigsten Genodermatosen im Universitätsspital Basel}

Die - Tab. 2 zeigt die 5 Genodermatosen, die am häufigsten im Universitätsspital Basel diagnostiziert wurden. Am meisten vertreten ist die Porokeratose mit $38 \%$ (43 Patienten), gefolgt von der Ichthyosis vulgaris mit 20,3\% (23 Patienten), dem Morbus Darier mit 15,0\% (17 Patienten), der Neurofibromatosis Recklinghausen mit 10,6\% (12 Patienten) und zuletzt der Epidermolysis bullosa mit 7,9\% (9 Patienten). Alle 5 genannten Genodermatosen werden autosomal-dominant vererbt, wobei in der Gruppe der Epidermolysen auch andere Vererbungswege existieren.

> Die 5 häufigsten Genodermatosen machen $91 \%$ der in der

Praxis zu beurteilenden genetischen Entitäten aus.
Tab. 3 Häufigkeitsverteilung der im Universitätsspital Basel diagnostizierten Genodermatosen

\begin{tabular}{|c|c|c|}
\hline Genodermatosen & Total & $\%$ \\
\hline & 210 & \\
\hline Erbliche Verhornungsstörungen & 100 & 47,6 \\
\hline Morbus Darier & 17 & \\
\hline Ichthyosis congenita, sonstige & 1 & \\
\hline Ichthyosis lamelläre & 4 & \\
\hline Ichthyosis vulgaris & 23 & \\
\hline Ichthyosis, X-chromosomale, rezessive & 2 & \\
\hline Keratoma palmare et plantare, hereditäres & 1 & \\
\hline Porokeratose & 43 & \\
\hline Porokeratosis superficialis disseminata actinica & 9 & \\
\hline Erbliche Epidermolysen & 24 & 11,4 \\
\hline Epidermolysis bullosa & 4 & \\
\hline Epidermolysis bullosa atrophicans generalisata gravis Herlitz & 1 & \\
\hline Epidermolysis bullosa dystrophica albopapuloidea Pasini & 1 & \\
\hline Epidermolysis bullosa dystrophica Hallopeau-Siemens & 2 & \\
\hline Epidermolysis bullosa dystrophica & 6 & \\
\hline Epidermolysis bullosa simplex & 9 & \\
\hline Epidermolysis bullosa, junktionale & 1 & \\
\hline Erbliche Bindegewebserkrankungen & 10 & 4,8 \\
\hline Cutis laxa & 1 & \\
\hline Ehlers-Danlos-Syndrom & 2 & \\
\hline Fokale dermale Hypoplasie, Goltz-Gorlin-Syndrom & 1 & \\
\hline Buschke-Ollendorff-Syndrom & 5 & \\
\hline Pseudoxanthoma elasticum & 1 & \\
\hline Ektodermale Dysplasien & 2 & 0,9 \\
\hline Dysplasie, ektodermale anhidrotisch & 1 & \\
\hline Dysplasie, ektodermale hidrotische & 1 & \\
\hline Erbliche Krankheiten der Haare oder der Nägel & 4 & 1,9 \\
\hline Hypotrichosis congenita & 3 & \\
\hline Trichothiodystrophie & 1 & \\
\hline Erbliche Pigmentstörungen & 5 & 2,4 \\
\hline Albinismus & 1 & \\
\hline Bloch-Sulzberger-Syndrom & 4 & \\
\hline Erbliche Stoffwechselstörungen & 13 & 6,2 \\
\hline Hereditäres angioneurotisches Ödem Quincke & 5 & \\
\hline Morbus Fabry, Angiokeratoma corporis diffusum & 2 & \\
\hline Porphyria cutanea tarda & 5 & \\
\hline Protoporphyria congenita & 1 & \\
\hline Genodermatosen mit Auftreten benigner Tumoren & 30 & 14,3 \\
\hline NAME-Syndrom & 2 & \\
\hline Neurofibromatose Typ 1 & 12 & \\
\hline Neurofibromatose, segmentale & 2 & \\
\hline Peutz-Jeghers-Syndrom & 2 & \\
\hline Sebocystomatosis Günther (Steatocystoma multiplex) & 1 & \\
\hline Epithelioma calcificans Malherbe & 4 & \\
\hline Epithelioma adenoides cysticum brooke & 1 & \\
\hline Epiloia, tuberöse Sklerose & 6 & \\
\hline Genodermatosen mit obligatem Auftreten maligner Tumoren & 11 & 5,2 \\
\hline Morbus Cowden & 1 & \\
\hline Basaliomatose, nävoide, Gorlin-Goltz & 4 & \\
\hline Epidermodysplasia verruciformis Lewandowsky-Lutz & 2 & \\
\hline
\end{tabular}


Tab. 3 Häufigkeitsverteilung der im Universitätsspital Basel diagnostizierten

Genodermatosen (Fortsetzung)

\begin{tabular}{lll}
\hline Genodermatosen & Total & $\%$ \\
\hline Xeroderma pigmentosum & 4 & \\
\hline Sonstige Genodermatosen & 11 & 5,2 \\
\hline Cockayne-Syndrom & 1 & \\
\hline Muckle-Wells-Syndrom & 2 & \\
\hline Rothmund-Thomson-Syndrom & 1 \\
\hline Werner-Syndrom & 1 \\
\hline Progerie & 1 \\
\hline Melkersson-Rosenthal-Syndrom & 2 \\
\hline Klippel-Trénaunay-Syndrom & 2 \\
\hline BK-Mole-Syndrom & 1 \\
\hline
\end{tabular}

\section{Porokeratose}

Die Diagnose der Porokeratosis erfolgt klinisch und histologisch (• Abb. 1). Es handelt sich um runde bis ovale, erythematöse Plaques mit einem scharfen hyperkeratotischen Randwall, die in jedem Alter neu auftreten können. In der disseminierten Form bevorzugen sie die Handrücken, Unterarmstreckseiten, Unterschenkel und Gesicht ohne Schleimhautbeteiligung. In der Histologie ist die kornoide Lamelle pathognomisch für alle Porokeratosisformen. Die kornoide Lamelle besteht aus einem nach außen ragenden Parakeratosekegel, dazwischen zeigen sich eine atrophe Epidermis sowie ein lymphozytäres Infiltrat. Bei segmentalen Formen vom Typ 2 der autosomal-dominant vererbten Dermatosen finden sich maligne Entartungen vorwiegend in den lineären Veränderungen durch einen Verlust der Heterozygotie [5].

\section{Ichthyosis vulgaris}

Die Diagnose erfolgt vor allem klinisch. Die Krankheit entwickelt sich im Verlauf des ersten Lebensjahres mit Hauttrockenheit und fein-lamellärer Schuppung. Vor allem sind die Streckseiten der Extremitäten sowie der Rumpf betroffen (• Abb. 2). Die Palmae und Plantae weisen eine etwas verdickte Haut mit vertieften Hautlinien auf. In 25\% der Fälle liegt begleitend eine atopische Diathese vor. Die Hautveränderungen sind in den trockenen Wintermonaten am stärksten ausgeprägt. In der Histologie zeigt sich im Stratum corneum eine Hyperkeratose, das Stratum granulosum ist reduziert bis fehlend. Das verantwortliche Gen Filaggrin ist mutiert und für die Krankheit ursächlich [6]. Eine molekulargenetische Diagnostik ist nicht notwendig.

\section{Darier-Krankheit}

Die Diagnose erfolgt sowohl klinisch als auch histologisch [7]. Die Erkrankung beginnt meist in der Pubertät mit follikulären und nicht follikulär gebundenen hyperkeratotischen bräunlichen Papeln in den seborrhoischen Arealen, die zeitweise auch rötlich bis grau sind und zu Plaques konfluieren können (• Abb. 3). Nägelmalformationen mit akralen Usuren und weißlichen Längsstreifen sowie Schleimhautbeteiligung finden sich oft. Eine Triggerung der Hautveränderungen ist durch UV-Exposition, Hitze und Schwitzen möglich. Histologisch zeigen sich eine säulenartige Hyperkeratose, eine Akanthose, eine Akantholyse mit „corps ronds“ im Stratum spinosum und "grains“ im Stratum granulosum und Stratum corneum sowie Lakunenbildungen. Die Krankheit wird durch eine Mutation in der Kalziumionenpumpe verursacht (ATP2A2). Molekulargenetik ist nicht erforderlich, da die Klinik und Histologie ausreichend für die Diagnosestellung sind.

\section{Neurofibromatose Typ I}

Die Diagnose erfolgt klinisch, wenn aus den folgenden Kriterien 2 oder mehr zutreffen: mindestens 6 Café-au-lait-Flecken, axilläre oder inguinale Pigmentierung („freckling“), mindestens 2 Neurofibrome oder ein plexiformes Neurofibrom, mindestens 2 Lisch-Knötchen, eine spezifische Knochenläsion, ein Verwandter ersten Grades mit peripherer Neurofibromatose, ein Optikusgliom (• Abb. 4). Die Pigmentflecken treten bereits ab Geburt oder in den ersten Lebensmonaten und Lebensjahren auf, Tumoren vor allem in der Pubertät. Die Neurofibrome zeigen sich als gestielte, weiche, hautfarbene bis rötlich-bräunliche Papeln. Tumore mit dem Risiko einer bösartigen Entartung, neurologische, orthopädische oder gravierende kosmetische Probleme stellen Gründe für eine operative Versorgung dar.

Die molekulargenetische Untersuchung erlaubt einerseits die Bestätigung der Diagnose bei Patienten mit insuffizienten klinischen Merkmalen, andererseits die Identifizierung der spezifischen Genmutation bei einem erkrankten Elternteil. Eine pränatale Diagnostik mittels Amniozentese oder Chorionzottenbiopsie ist prinzipiell möglich. Die stark variierende Ausprägung der Erkrankung, die vielfältigen Symptome sowie die unterschiedlichen Verläufe erschweren die Erarbeitung einer Prognose bezüglich des Schweregrades der Krankheit.

\section{Epidermolysis bullosa}

Die exakte Diagnose erfolgt klinisch und histologisch sowie molekulargenetisch. Die Patienten leiden unter Bildung von Blasen, die nach mechanischer Belastung entstehen und entweder mit oder ohne Atrophie bzw. Narbenbildung abheilen (- Abb. 5). Die Epidermolysis simplex heilt narbenlos ab, da sie intraepithelial gelegen ist, die junktionale und dystrophe Form sind vernarbend. Die Hautveränderungen können sich bereits ab der Geburt oder erst im Erwachsenalter bemerkbar machen. Wichtig ist hier die Beobachtung des Verlaufs. Die Hautbiopsien mit SkinSplit-Methode dienen der sicheren Diagnose und der Unterscheidung der Epidermolyis-bullosa-Typen. Sie werden mittels Elektronenmikroskopie und Immunfluoreszenzmikroskopie untersucht und letztlich molekulargenetisch bestimmt.

Die Immunfluoreszenzmikroskopie ermöglicht dank der Technik des Antigenmappings sowohl die Unterscheidung der Blasenbildungsebene als auch die Ge- 
winnung von Erkenntnissen auf molekulargenetischer Ebene [8].

Mittels Molekulargenetik wird das mutierte Gen beim Patienten nachgewiesen, was eine pränatale Diagnostik mit konsekutiver genetischer Beratung im Rahmen der Familienplanung möglich macht.

Im Allgemeinen arbeitet die medizinische Genetik des Universitätsspitals Basel mit allen Genetikinstituten der Schweiz sowie mit Instituten im Ausland zusammen, abhängig von der konkreten Fragestellung. Die Neurofibromatosis Typ 1 wird z. B. am Inselspital Bern, Molekulargenetik, untersucht; die Epidermolysis bullosa molekulargenetisch vorwiegend an der Universität Freiburg im Breisgau, wobei die medizinische Genetik in Basel gewisse Gene/Exons mittlerweile ins Repertoire aufgenommen hat.

\section{Diskussion}

In unserer Studie wurden bei 210 Personen von 48.354 Patienten der dermatologischen Universitätsklinik Basel 50 verschiedene Genodermatosen diagnostiziert. Dies entspricht weniger als $1 \%$ des Patientenkollektivs in der Zeitspanne von 2002 bis 2008 im Universitätsspital Basel. Diese 210 Patienten gehören wiederum zu jenen 30 Mio. Personen, die in Europa von einer seltenen Erkrankung betroffen sind („Orphan diseases“). Es scheint also vorerst eine kleine Gruppe Erkrankter zu sein, doch in Wirklichkeit ist die Anzahl der Betroffenen viel höher. Zunehmende molekulargenetische diagnostische Möglichkeiten und auch vermehrte Therapieoptionen werden diese Patienten in steigendem Maß in die Praxis der Niedergelassenen und Universitätsspitäler bringen.

\section{I) Die erblichen}

\section{Verhornungsstörungen werden am häufigsten diagnostiziert}

In unserem Kollektiv wurden die erblichen Verhornungsstörungen am häufigsten diagnostiziert, was ebenfalls der Häufigkeitsverteilung in der Literatur entspricht. Drei Krankheiten unter unseren 5 häufigsten Genodermatosen, die Ichthyosis vulgaris, die Porokeratosis sowie die
Darier-Krankheit, gehören dieser Gruppe an.

Die Porokeratosis wird als relativ häufige Genodermatose beschrieben, wobei keine Zahlen zu der Inzidenz zu finden sind. In unserem Kollektiv war die Porokeratosis die am meisten diagnostizierte Genodermatose mit 9/10.000 dermatologischen Patienten. Die Ichthyosis vulgaris, die als häufigste Genodermatose überhaupt gezählt wird, kam in unserer Studie als zweithäufigste vor. Im Vergleich zur Inzidenz in der Literatur wurde sie bei uns ca. 8-mal weniger häufig diagnostiziert. Diese Entwicklung kann damit im Zusammenhang stehen, dass bei einer milden Verlaufsform die Ichthyosis vulgaris unentdeckt bleiben kann und es somit gar nicht zur Diagnose kommt.

Die Inzidenz der Darier-Krankheit in unserem Kollektiv entspricht beinahe jener der Literatur mit 3,5/10.000. Die Neurofibromatose Typ 1 wurde weniger häufig diagnostiziert, während die Epidermolysis bullosa bei uns zwischen 8und 16-mal öfter festgestellt wurde. Dies könnte dafür sprechen, dass das Universitätsspital Basel vermehrt als Referenzzentrum für Genodermatosen fungiert, und dementsprechend mehr Patienten mit Verdacht auf eine genetisch vererbte Hauterkrankung der dermatologischen Klinik zugewiesen werden.

\section{Fazit für die Praxis}

- Genodermatosen sind selten, doch es gibt außerordentlich viele verschiedene und sehr seltene genetische Erkrankungen der Haut, sodass der Praktiker diese nicht alle kennen kann.

- Unsere epidemiologische Untersuchung hat gezeigt, dass folgende 5 Genodermatosen $91 \%$ aller Besuche von Patienten mit genetischen Hauterkrankungen ausmachen: Porokeratosen, Ichthyosis vulgaris, Morbus Darier, Neurofibromatose Recklinghausen und Epidermolysis bullosa. Es lohnt sich also, diese Entitäten zu kennen.

- Die wichtigste Datenbank für monogene Erkrankungen ist die OMIM-
Website, die auch für den Praktiker hilfreiche Informationen enthält.

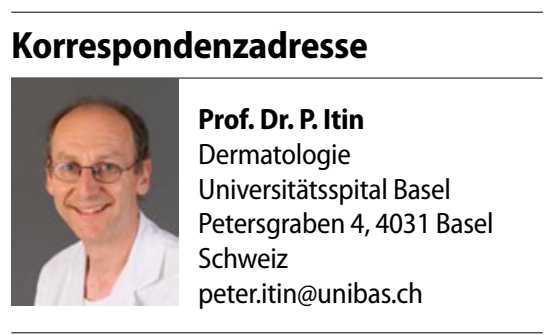

Interessenkonflikt. Der korrespondierende Autor gibt für sich und seinen Koautor an, dass kein Interessenkonflikt besteht.

\section{Literatur}

1. Itin PH, Burger B (2010) Genodermatoses for practitioners - principles and concepts. Ther Umschau 67:483-485

2. Manolio TA, Brooks LD, Collins FS (2008) A HapMap harvest of insights into the genetics of common disease. J Clin Invest 118:1590-1605

3. Feramisco JD, Sadreyev RI, Murray ML et al (2009) Phenotypic and genotypic analyses of genetic skin disease through the Online Mendelian Inheritance in Man (OMIM) database. J Invest Dermatol 129:2628-2636

4. Amberger J, Bocchini C, Hamosh A (2011) A new face and new challenges for Online Mendelian Inheritance in Man (OMIM $\left.{ }^{\circledast}\right)$. Hum Mutat 32:564567

5. Happle R (1997) Cancer proneness of linear porokeratosis may be explained by allelic loss. Dermatology 195:20-25

6. Brwon SJ, McLean WH (2012) One remarkable molecule: filaggrin. J Invest Dermatol 132:751-762

7. Itin P (2011) Darier disease (Darier-White disease). In: Schachner LA, Hansen RC (Hrsg) Pediatric dermatology. Edinbourgh, Mosby, S 636-639

8. Intong LR, Murrell DF (2012) Inherited epidermolysis bullosa: new diagnostic criteria and classification. Clin Dermatol 30:70-77 